\title{
Digital equalization of time-delay array receivers on coherent laser communications
}

\author{
ANicETO BeLmonte \\ Technical University of Catalonia, BarcelonaTech, Dept. of Signal Theory and Communications, 08034 Barcelona, Spain \\ belmonte@tsc.upc.edu
}

\begin{abstract}
Field conjugation arrays use adaptive combining techniques on multi-aperture receivers to improve the performance of coherent laser communication links by mitigating the consequences of atmospheric turbulence on the down-converted coherent power. However, this motivate the use of complex receivers as optical signals collected by different apertures need to be adaptively processed, co-phased, and scaled before they are combined. Here we show that multiple apertures coupled with optical delay lines combine retarded versions of a signal at a single coherent receiver, which uses digital equalization to obtain diversity gain against atmospheric fading. We found in our analysis that, instead of field conjugation arrays, digital equalization of time-delay multi-aperture receivers are a simpler and more versatile approach to accomplish reduction of atmospheric fading.
\end{abstract}

\section{(C) 2016 Optical Society of America}

OCIS codes: (060.1660) Coherent communications; (060.2605) Freespace optical communication; (040.1240) Arrays, apertures

http://dx.doi.org/10.1364/OL.99.099999

Coherent laser communications through the atmosphere are challenging because turbulence disturbs the received optical wavefront and their mixing with the local oscillator [1,2]. A coherent fiber array consisting of an assembly of smaller fiber-coupled apertures can be used to replace a single monolithic-aperture receiver with a full-size collecting area. Output signals from the optical fibers are combined optically in a fiber power combiner and, as the array output is accessible in a single fiber, superimposed with the local oscillator in a directional coupler. The receiver uses balanced detection so the down-converted electrical signal can be measured coherently.

Since each receiver aperture in a coherent fiber array can be smaller than the scale on which the optical wavefront fluctuates, a coherent fiber array has a gain in terms of coupling efficiency because the optical signal can be matched with less error to the propagating mode of a single-mode fiber. In general, given a fixed collecting area, the performance of the array should improve with an increasing number of apertures [3-6].

Nevertheless, the atmospheric optical signals received by the different apertures in the array exhibit field fading, i.e., random fluctuations of both envelope and phase over time. Fading will cause destructive interference in the optical combiner and reduce the strength of the total optical signal [3]. Assuming that the dominant noise $n(t)$ source is shot noise from the local oscillator laser, which can be modeled accurately as additive white Gaussian noise (AWGN) that is statistically independent of the atmospheric fading, the down-converted detected array signal $r_{a}(t)$ can be written as

$$
r_{a}(t)=\sum_{l=0}^{L-1}\left[\alpha_{l} s(t)\right]+\mathrm{n}(t) .
$$

Here, $\alpha_{l}=\left|\alpha_{l}\right| \exp \left(j \phi_{l}\right)$ denotes the atmospheric fading at aperture $l \in\{0,2, \ldots, L-1\} \in \mathbb{N}^{L}$, where $\left|\alpha_{l}\right|$ represents the fading envelope and $\phi_{l}$ the corresponding random phase of the optical signal. The transmit signal waveform $s(t)=\sum_{n} d(n) p(t-n T)$ for the data symbol sequence $d(n), n \in\{1,2, \ldots, N\} \in \mathbb{N}^{N}$ is the sum of pulses with shape $p(t)$ transmitted per symbol interval $T=1 / B$, when $B$ is the signal spectral bandwidth. For a shotnoise-limited array receiver, the composite signal-to-noise ratio (SNR) per symbol $\gamma=\gamma_{0} \alpha^{2}$ can be taken as the number of signal photons received on the receiver multiaperture $\gamma_{0}$ multiplied by a mixing efficiency $\alpha^{2}=$ $\left|\sum_{l} \alpha_{l}\right|^{2}$.

Fading on the array $L$ receive apertures can be aggregated into a complex channel column vector $\boldsymbol{\alpha}=$ $\left(\alpha_{0}, \alpha_{2}, \ldots, \alpha_{L-1}\right)^{T} \in \mathbb{C}^{L}$, where the superscript ${ }^{\mathrm{T}}$ denotes transposition. It is recognized [6] that, if instantaneous atmospheric fading information $\boldsymbol{\alpha}$ is known for all apertures in Eq. (1), an adaptive linear combiner $\boldsymbol{w}=\left(w_{1}, w_{2}, \ldots, w_{L}\right)^{\boldsymbol{T}} \in \mathbb{C}^{\boldsymbol{L}}$ can be considered to compensate for fading effects and match the coherent array to the optical input field (see Fig. 1(a)). When a linear combiner is considered along with the array, the receive signal $r_{c}(t)$ is the result of adding together the scaled and phase shifted signals received from the various receive apertures:

$$
r_{c}(t)=\sum_{l=0}^{L-1}\left[w_{l} \alpha_{l} s(t)\right]+\mathrm{n}(t) .
$$

Here, the complex weight $w_{1}$ of the linear combiner applied to the $l$ th subaperture output can be characterized at large as $w_{l}=\left|w_{l}\right| \exp \left(j \theta_{l}\right)$ where $\left|w_{l}\right|$ and $\theta_{l}$ are the 
amplitude and phase controls, respectively, provided by the linear combiner. Field conjugation array combining $\boldsymbol{w}=1 / \boldsymbol{\alpha}^{*}$ makes maximal-ratio combining possible and produces perfect mixing of the array signals. Now, the resultant composite SNR of the envelope detector for an $L$ element combiner is the sum of the component array element SNR's, i.e., $\gamma=\gamma_{0} \sum_{l}\left|\alpha_{l}\right|^{2}$ and a fraction equal to $\left|\alpha_{l}\right|^{2}$ of the incident received photons $\gamma_{0}$ per symbol are coupled into the $l$ th aperture.

However, the conjugate array described by Eq. (2) requires the use of complex receivers as atmospheric fading information $\alpha_{l}=\left|\alpha_{l}\right| \exp \left(j \phi_{l}\right)$ needs to be estimated for all apertures. Also, optical signals collected by different apertures need to be adaptively processed, $\mathrm{co}^{-}$ phased, and scaled with the complex weight $w_{l}=$ $\left|w_{l}\right| \exp \left(j \theta_{l}\right)$ before they are combined. Phase $\theta_{l}$ and amplitude $\left|w_{l}\right|$ control is usually achieved by introducing an optical phase shifter and an optical amplifier stage after each aperture in the array.

Here we show a simpler and more versatile array approach to accomplish reduction of atmospheric fading (Fig. 1(b)). The diversity scheme introduced in this work converts spatial diversity into delayed signals and superposes in the span of a symbol $T$ the equivalent to $L$ atmospheric fading realizations. The scheme considers a fiber array where each aperture feeds a single-mode fiber and where the fields of each aperture are properly delayed controlling the length of each fiber (performing as optical delay lines). Each succeeding optical fiber in the array needs to be larger than the preceding by an additional length of $\mathrm{v} T$ meters, with $\mathrm{v}$ the group velocity of light in fiber. For instance, in a 1550-nm wavelength system, where $v$ is roughly $2 / 3$ of light speed in a vacuum, a $10^{-}$ GBd data rate, with a symbol time frame $T$ of $0.1-\mathrm{ns}$, requires a relative additional length $v T$ of $2 \mathrm{~cm}$. We note that, although other devices could be used to symbolspaced delay the optical signals in this diversity scheme, fiber optics are attractive delay lines due to its flexibility and low propagation losses.

Now, no adaptive linear combiner $\boldsymbol{w}$ is considered in the proposed scheme and the receive signal is generated from the symbol-spaced $T$ delay superposition and interference of the signals received from the $L$ receive apertures, i.e., $\alpha_{l} s(t-l T)$. The signal $r(t)$ received by the time-delay array is given by

$$
r(t)=\sum_{l=0}^{L-1}\left[\alpha_{l} s(t-l T)\right]+n(t) .
$$

Here, along with the fading effects $\alpha_{l}$, the overlay at the receiver of multiple delayed copies of the transmitted signal $s(t-l T)$ creates an artificial intersymbol interference (ISI). This is a unique feature of the proposed time-delay scheme. When no delayed signals are considered, intersymbol interference between two symbol transmission is negligible in free-space optical channels due to extremely small multi-path effects in the atmosphere.

It is well known that the effects of multipath ISI in RF wireless systems can be reduced using transmit diversity and equalization [7]. In this analysis we use receive diversity and signal equalization to mitigate the effects of both ISI and atmospheric fading in Eq. (3). The goal is for the combination of fading, symbol interference, and equalization to provide an enhanced received signal [8].

The process of equalizing to mitigate interference effects involves using digital methods to gather the dispersed symbol energy $\alpha_{l} s(t-l T), l \in\{0,2, \ldots, L-1\}$ in Eq. (3) back into its original time interval $s(t)$ so that it doesn't complicate the detection of other symbols. Simultaneously, a digital equalizer also provide fading diversity by synthetizing an inverse filter of the atmospheric fading channel $\boldsymbol{\alpha}$ and applying it to the different components $\alpha_{l} s(t), l \in\{1,2, \ldots, L\}$ of the gathered dispersed signal. Since atmospheric signal distortions can be expressed as linear transfer functions operating on the complex amplitude of the optical signal, in principle we can compensate for them by linearly equalizing the coherent-detected complex amplitude with digital techniques.

A simple means of implementing such a filter in our diversity scheme is the use of a symbol-spaced adaptive linear equalizer (LE) [9], where the digital equalized signal $y[n]=y(n T), n \in\{1,2, \ldots, N\}$ can be obtained by sampling the output signal $r(t)$ at the symbolsynchronous intervals. This class of equalizer is called symbol-spaced because the sample rates of the input and output are equal. The digital observations $y[n]$ can be written as

$$
y[n]=\sum_{l=0}^{L-1} c_{l} r(n T-l T) .
$$

Here, a set of weighting coefficients $\boldsymbol{c}=\left(c_{0}, c_{2}, \ldots, c_{L-1}\right)^{T} \in \mathbb{C}^{L}$, describing the taps of a linear finite impulse response (FIR) filter, is used to compensate for intersymbol interference and atmospheric fading effects in the received signal $r(t)$. The number of FIR taps in Eq. (4) equals the number of apertures $L$ and the $l$ th tap implements a temporal delay. The binary data sequence $d(n)$ is detected from this digital signal $y[n]$ and the equalization technique works well because the coefficients $c_{l}$ are determined from training patterns affected by the atmospheric impairments. The coefficients $\boldsymbol{c}=\left(c_{0}, c_{2}, \ldots, c_{L-1}\right)^{T}$ of the equalizer can be adapted to the time-varying atmospheric channel characteristics using a variety of methods such as the least-mean square (LMS) algorithm. It is worth mentioning that, as optical signal rates $1 / T$ increase up to several $\mathrm{GHz}$, the atmospheric channel remains constant over a coherence time $\tau$ much larger than the transmission duration of the symbol $T$. Atmospheric turbulent fluctuates with a rate $1 / \tau$ that is generally no higher than $1 \mathrm{kHz}$.

Among all digital equalization techniques, decision feedback equalizers (DFE) and maximum-likelihood sequence estimation equalizer (MLSEE) can be considered as natural extensions of LE in Eq. (4). DFE depends on the idea that, once you have determined the value of the current transmitted symbol, you can exactly remove the intersymbol interference contribution of that symbol to future received symbols [9]. DFE consists of an LE with an additional filter to process past symbols 
(a)

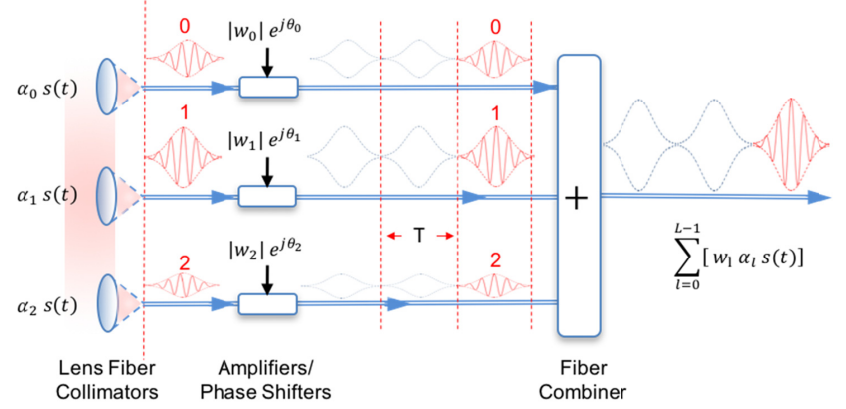

(b)

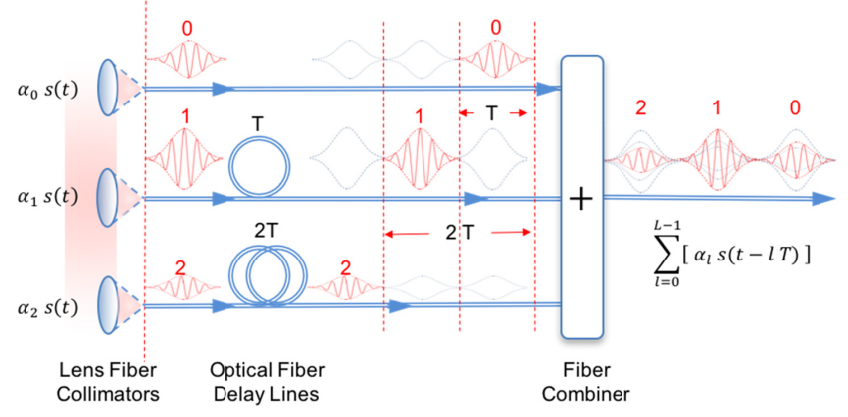

Fig. 1. In free-space laser communications, coherent diversity receivers implemented using $L$ multiple optical apertures are used to mitigate atmospheric fading. To keep the figure simple, only 3 apertures are shown. (a) Field conjugation arrays use adaptive combining techniques to mitigate fading on the down-converted coherent power (b) The proposed scheme converts spatial diversity into delayed signals and superposes in the span of a symbol $T$ the equivalent to $L$ atmospheric fading realizations. The overlay at the receiver of multiple delayed copies of the transmitted signal creates intersymbol interference (ISI). After the coherent receiver, an analog-to-digital A/D converter is used in conjunction with digital signal processing (DSP) manipulation (digital equalizer).

decisions $d(n)$ in order to cancel any remaining ISI. On the other hand, MLSEE invokes the optimum receiver in terms of minimizing the data sequence error rate [10]. Given a set of $N$ observations $\mathrm{y}[n]$, the likelihood function $\mathcal{L}(\mathrm{d}(n) \mid \mathrm{y}[n])$ is maximized by the most likely transmitted data sequence $\mathrm{d}(n)$. The optimal transmitted sequence

$\mathrm{d}(n)$ consistent with the observations $\mathrm{y}[n]$ can be solve recursively by using the Viterbi algorithm [11]. We note that the complexity of the Viterbi algorithm increases with the number of interfering symbols and taps $L$. As a consequence, MLSEE practical use is limited to array receivers with a not excessive number of apertures $L$. On the other hand, DFE has a computational complexity which grows linearly with the number of taps $L$ and may be more convenient when very large aperture arrays are needed.

Without loss of generality, in this work we consider the performance of BPSK modulation using coherent detection in the presence of AWGN and atmospheric fading. We illustrate the performance offered by the proposed diversity scheme on free-space laser communications when the three main types of equalizers -i.e., LE, DFE, and MLSEE- are implemented in the digital receiver and confront typical atmospheric channel conditions. We have conducted a numerical simulation analysis and a set of experiments is performed on synthetically built array signals. For comparison of the receiver performance between an $L$-element array and a single large aperture, we force each subaperture in the array to have a pupil area equal to $1 / L$ times the pupil area of the single receiver system.

In order to synthesize the array signals, we consider the statistical properties of the atmospheric channel [3, 12] and simulate the corresponding fade $\boldsymbol{\alpha}=$ $\left(\alpha_{0}, \alpha_{2}, \ldots, \alpha_{L-1}\right)^{T}$. The strongest turbulence level used in this analysis is quantified by a normalized aperture diameter $D / r_{0}=10$ and a scintillation index $\sigma_{\beta}^{2}=0.3$. Here, $D$ is the aperture diameter of the single receiver system and the coherence diameter $r_{0}$ describes the spatial correlation of phase wavefront fluctuations in the receiver plane [13]. For a fixed aperture diameter $D$, the photoelectric down-conversion efficiency reduces with decreasing coherent diameter $r_{0}$. The scintillation index $\sigma_{\beta}^{2}$, describing wave-front amplitude fluctuations, is set to a level below the saturation regime [14].

Figure 2 considers symbol error rate (SER) as a function of the total number of signal photons $\gamma_{0}$ collected on the array multi-aperture when digital equalization techniques are used. In all graphics, the corresponding curves without diversity (single-aperture) and without fading (AWGN limit) are shown for reference.

Figure 2(a) compares the performance of the three different equalizers when used in conjunction with a 13element array. We consider a 1550-nm, 10-GBd system and use data sequences $d(n)$ of length $N=2048$ symbols. It is apparent from the plots that the LE filter does not perform well and, at best, it is only able to offer a mere $3-\mathrm{dB}$ diversity gain against the strong atmospheric fading considered here $\left(D / r_{0}=10\right)$. As expected, the DFE filter fares much better and reach a diversity gain higher than $10 \mathrm{~dB}$ at $10^{-3}$ symbol error rate. Finally, the more complex MLSEE shows almost optimal performance. A moderate-size 13-element array receiver, which is easily handled by the MLSEE filter, produces a large 14- $\mathrm{dB}$ diversity gain at $10^{-3}$ SER. For comparison, the corresponding results for an ideal 13-branch fieldconjugation array receiver (see Eq. (2) above) is given. It shows how multi-aperture receiver systems with optically delayed receive signals and MLSEE filtering provides a diversity gain within a fraction of $\mathrm{dB}$ of that with complex receive field-conjugation arrays. The optimum MLSE receiver emulates digitally the atmospheric channel and synthetize the optimal inverse filter $1 / \boldsymbol{\alpha}^{*}$, providing ideal fading diversity to the different components $\alpha_{l} s(t), l \in$ $\{1,2, \ldots, L\}$ of the collected signal.

We see in the plots that it requires more than $20-\mathrm{dB}$ SNR $\gamma_{0}$ per symbol to maintain a $10^{-3}$ SER in atmospheric fading (single-aperture). This represents a significant $15-\mathrm{dB}$ SNR fading penalty with respect to the AWGN limit. Figure 2(b) considers the MLSEE performance for different number of array apertures $L$. 

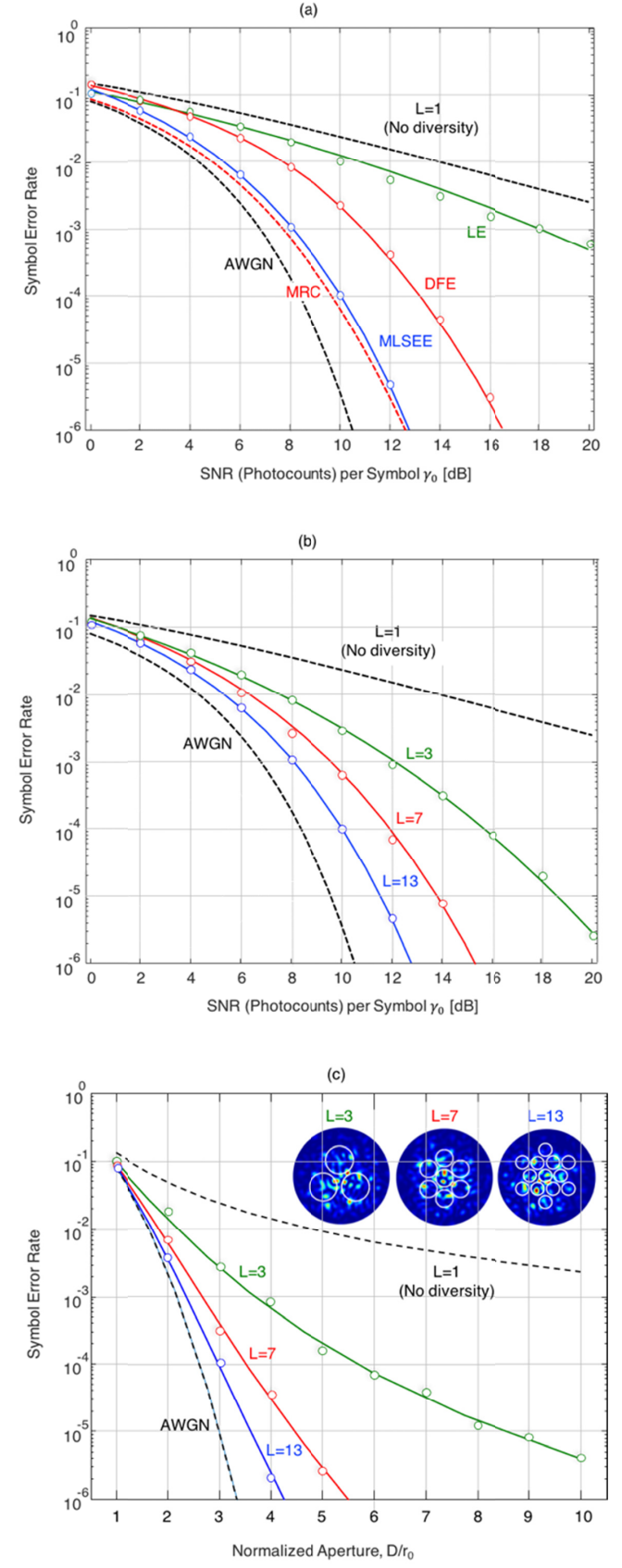

Fig. 2. SER for BPSK modulation as a function of the number of signal photons (SNR) per symbol $\gamma_{0}$ collected on the array aperture. (a) SER measurements compare the performance of LE, DFE, and MLSE equalizers with a 13-element optical array. For comparison, the corresponding result for a perfect 13-aperture maximal-ratio array combiner (MRC) is given. (b) SER for different numbers of apertures $L$ in the array receiver when a MLSEE is considered. (c) SER performance for the MLSEE is presented as a function of the normalized aperture $D / r_{0}$ for a constant phase coherence length $r_{0}$. The smallest aperture considered in the plots assumes $\gamma_{0}$ equal to 1 photons-per-symbol and, for any aperture diameter, the value of $\gamma_{0}$ increases with $D^{2}$. In all graphics, the corresponding curves without diversity $(L=1)$ and without fading (AWGN limit) are shown for reference. In (c) (inset), the arrays composed of $L=3,7$, and 13 receive apertures considered in this analysis.

SER is considered for systems with 3,7 , and 13 apertures. After equalization of the delayed signals collected by just three apertures, the system demands 12$\mathrm{dB}$ SNR $\gamma_{0}$ to maintain the $10^{-3}$ SER, which still represents a $5-\mathrm{dB}$ penalty with respect to the AWGN. However, for 7-aperture and 13-aperture array receivers, the SNR penalty reduces to $3 \mathrm{~dB}$ and $1 \mathrm{~dB}$, respectively.

Figure 2(c) considers the effect of aperture diameter on the MLSEE performance. It presents the SER as a function of the normalized aperture $D / r_{0}$ for a constant phase coherence length $r_{0}$. The SER depends on both the number of signal photons received on the receiver multiaperture $\gamma_{0}$ and the mixing efficiency $\alpha^{2}$ through the composite signal-to-noise ratio (SNR) per symbol $\gamma=\gamma_{0} \alpha^{2}$. The aperture diameter $D$ affects $\gamma_{0}$ and $\alpha^{2}$ inversely. For any aperture diameter, the value of $\gamma_{0}$ increases with $D^{2}$. Simultaneously, for a fixed coherent diameter $r_{0}$, as the aperture diameter $D$ is increased, the normalized aperture diameter $D / r_{0}$ increases, and turbullence reduces the downconversion mixing efficiency $\alpha^{2}$. When $L=1$, the system reaches a minimum $2 \times 10^{-3}$ SER when the normalized aperture is $D / r_{0}=10$. As expected, an increase in the number of array subapertures will improve the situation and decrease the SER.

In summary, we described in our analysis how multiple apertures coupled with optical delay lines combine retarded versions at a single coherent receiver, which uses digital equalization to obtain diversity gain against atmospheric fading. We discussed the average symbol error rate as a measure of performance and pondered several potential digital equalization structures. In particular, the results of our analysis showed that multiaperture receiver systems with MLSEE filters provide a diversity gain analogous to the gain of the perfect field conjugation array combiner. All in all, the digital equalizer seemed to act in the temporal domain as the field conjugation combiner does in the space domain by digitally providing the receiver with symbol energy that would otherwise be lost to interference. This research opens new possibilities for digital signal processing techniques to enhance free-space coherent optical communications.

\section{References}

1. A. Belmonte and J. M. Kahn, Opt. Express 16, 14151-14162 (2008).

2. I. S. Ansari, F. Yilmaz, and M. S. Alouini, in 81 st Veh. Technol. Conf. (IEEE, 2015), pp. 1-5.

3. A. Belmonte and J. M. Kahn, Opt. Express 17, 12601-12611 (2009).

4. E. J. Lee and V. W. Chan, J. Opt. Commun. Netw. 1, 463-483 (2009).

5. M. Niu, J. Cheng, and J. F. Holzman, Opt. Express 18, 13915-13926 (2010).

6. A. Belmonte and J. M. Kahn, J. Lightwave Technol. 31, 1383-1387 (2013).

7. J. H. Winters, IEEE Trans. Veh. Technol. 47, 119-123 (1998).

8. J. G. Proakis and M. Salehi, Digital Communications, (Mc Graw-Hill, 2007).

9. S. Qureshi, Proc. IEEE 73, 1349-1387 (1985).

10. G. D. Forney, IEEE Trans. Inf. Theory 18, 363-378 (1972).

11. G. D. Forney, Proc. IEEE 61, 268-278 (1973).

12. A. Belmonte, J. Lightwave Technol. 32, 2128-2132 (2014).

13. D. L. Fried, Proc. IEEE 55, 57-67 (1967).

14. L. C. Andrews and R. L. Phillips, Laser Beam Propagation Through Random Media (SPIE Press, 2005). 


\section{References}

1. A. Belmonte and J. M. Kahn, "Performance of synchronous optical receivers using atmospheric compensation techniques," Opt. Express 16, 14151-14162 (2008).

2. I. S. Ansari, F. Yilmaz, and M. S. Alouini, "Performance Analysis of FSO Links over Unified Gamma-Gamma Turbulence Channels," in 81st Veh. Technol. Conf. (IEEE, 2015), pp. 1-5.

3. A. Belmonte and J. M. Kahn, "Capacity of coherent free-space optical links using diversity-combining techniques," Opt. Express 17, 12601-12611 (2009).

4. E. J. Lee and V. W. Chan, "Diversity Coherent and Incoherent Receivers for Free-Space Optical Communication in the Presence and Absence of Interference," J. Opt. Commun. Netw. 1, 463-483 (2009).

5. M. Niu, J. Cheng, and J. F. Holzman, "Exact error rate analysis of equal gain and selection diversity for coherent free-space optical systems on strong turbulence channels," Opt. Express 18, 13915-13926 (2010).

6. A. Belmonte and J. M. Kahn, "Sequential Optimization of Adaptive Arrays in Coherent Laser Communications," J. Lightwave Technol. 31, 1383-1387 (2013)

7. J. H. Winters, "The diversity gain of transmit diversity in wireless systems with Rayleigh fading," IEEE Trans. Veh. Technol. 47, 119-123 (1998).

8. J. G. Proakis and M. Salehi, Digital Communications, (Mc Graw-Hill, 2007).

9. S. Qureshi, "Adaptive equalization," Proc. IEEE 73, 1349-1387 (1985).

10. G. D. Forney, "Maximum likelihood sequence estimation of digital sequences in the presence of intersymbol interference," IEEE Trans. Inf. Theory 18, 363-378 (1972).

11. G. D. Forney, "The Viterbi algorithm," Proc. IEEE 61, 268-278 (1973).

12. A. Belmonte, "Capacity of coherent laser downlinks," J. Lightwave Technol. 32, 2128-2132 (2014)

13. D. L. Fried, "Optical heterodyne detection of an atmospherically distorted signal wave front," Proc. IEEE 55, 57-67 (1967).

14. L. C. Andrews, R. L. Phillips, Laser Beam Propagation Through Random Media (SPIE Press, 2005). 